\title{
OENORM B4014/2 - New Austrian code on dynamic wind loads: A step towards realistic modelling of wind loads in engineering applications
}

\author{
Helmut Sockel ${ }^{* 1}$ Peter H.W. Prenninger*2
}

\section{SUMMARY}

The basic concept and content of the new Austrian code on dynamic wind loads OENORM B4014/2 is discussed in this paper. Furthermore, the application of this code is demonstrated by analyzing the wind loadings of two typical sample structures. Finally, these results are compared with the wind loads determined according to the Japanese code presently in use and other corresponding codes. The results of this comparative study indicate a good agreement between the wind loads obtained from both the Austrian and Japanese code.

\section{INTRODUCTION AND HISTORICAL BACKGROUND}

Until some decades ago, civil engineering structures were only of moderate height, the constructions were rigid and the roof angles high. Based on the experience with structures like these, the rules in the codes were defined. But the constructions changed, the height of the structures increased and at the same time the weight decreased, the structures became more flexible. The roof angles decreased, many buildings were fitted with flat roofs. But the calculation of the wind loads was done with rules of the code based on the experience with the old structures.

Because of the occurence of structural failures, model experiments in wind tunnels and theoretical investigations were performed in order to dedect the reason for the failure of the structures. The engineering science made significant progress in the ability of predicting wind effects on buildings and structures. Consequently, it was time to revise the codes.

The old Austrian code published first in 1955 was valid until

*1 Inst. of Fluid Mechanics and Heat Transfer, Technical Univ. of Vienna, Austria;

*2 Dept. of Civil Eng., Kyoto Univ., on leave from Inst. of Engineering. Mechanics, Univ, of Innsbruck, Austria 
1980 with some minor modifications. In this old code we find that an act of god can be assumed, if there is a structural failure at a wind speed exceeding $90 \%$ of speed given in the code. But results of experiments have shown that there is no higher power causing the failures, so that the code proved to be insufficient. Hence, the work of revision was started at the beginning of the seventies.

The old Austrian code for wind loads and forces due to earthquake consisted of five pages. The practical engineers wanted a new code, but they wanted one as short and as simple as the old one. So the discussions lasted for years until 1980 when the first part of the new Austrian wind code (OENORM B4014/1) including the rules for structures non susceptible to vibrations were published. The work on the second part (OENORM B4014/2) concerning wind loads on structures susceptible to vibrations was finished this year and will now be published for comments.

\section{BASIC CONCEPT OF WIND LOAD CODIFICATION IN AUSTRIA}

As mentioned in the introduction the application of advanced analytical methods in combination with new, high strength materials enables the design of tall structures with significantly increased sensitivity for wind induced vibrations. Especially decreasing eigenfrequencies of such structures can lead to strong resonance effects due to dynamic wind loadings. These dynamic wind load mechanisms cannot correctly be described with equivalent static loads without considering the dynamic characteristics of the structure analyzed. On the other hand, of course, there is still the large group of wind loaded structures which eigenfrequencies are located at a very high frequency range compared to the characteristic frequencies of the dynamic wind loads. This class includes e.g. low rise buildings and short span bridges. The wind loads excerted on such structures can be characterized with equivalent quasistatic forces. Obviously the description of these equivalent static forces has not to include information corresponding to the dynamic behaviour of the structures and, therefore, can easily be included in wind load codes.

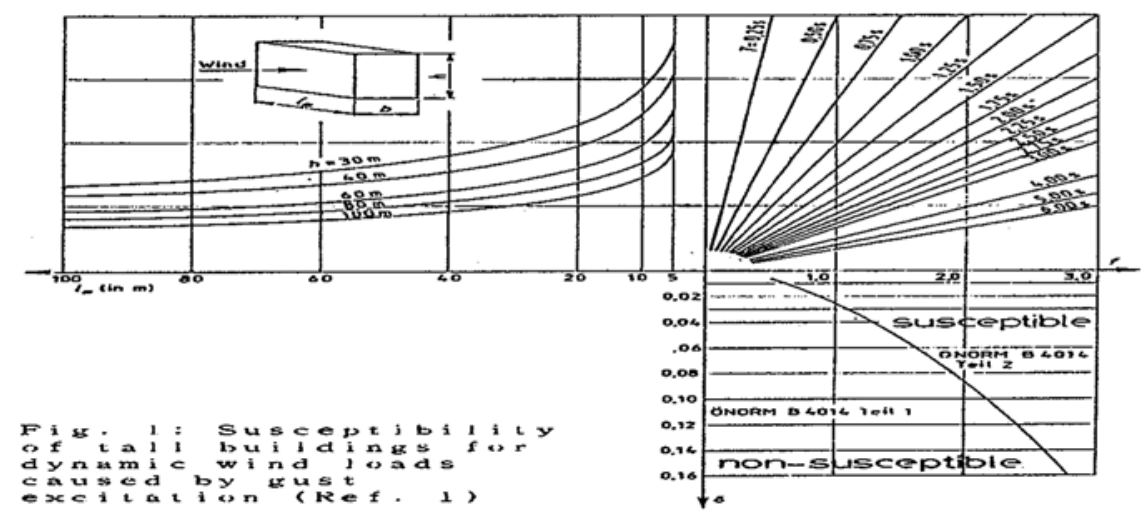

Because of this, the recent version of the first part of the Austrian code OENORM B4014/1 already includes a classification procedure in order to provide a tool for the designing engineer 
to decide whether a structure has to be considered as susceptible or not for dynamic wind loadings due to gust excitation. This classification is based on an empirical equation (Ref. 3) as shown below.

$$
\mathrm{f}=\mathrm{T}\left(\mathrm{v}_{\mathrm{o}} / \mathrm{h}\right)\left(\mathrm{h} / \mathrm{l}_{\mathrm{m}}\right)^{1 / 3}\left(\mathrm{~h} / \mathrm{h}_{\mathrm{o}}\right)^{1 / 7}
$$

where $f$ is a susceptibility factor for dynamic wind loads, $T$ the natural period of the structure in $s, v_{0}$ and $h_{0}$ constants with the values of $25.3 \mathrm{~m} / \mathrm{s}$ and 10 . m, respectively, and $\mathrm{h}$ is the total height of the structure. By taking into account the logarithmic damping decrement of the structure and the factor f from the equation above, the susceptibility of a particular structure can be decided based on graph as shown in Fig. 1.

Besides the structures identified with Equ. 1 to be susceptible for dynamic wind loading, generally, all structures exceeding the height of $100 \mathrm{~m}$ or with an aspect ratio greater than 20 should be designed considering the dynamic action of wind loads.

For the lack of simplicity in Fig. 1 a nomogram is included which graphically shows the respective relations expressed by Equ. 1. From this equation as well as the nomogram it can be seen that geometrical parameters such as height as well as width and structural parameters such as lowest eigenfrequency and damping are considered in order to decide whether or not dynamic wind effects have to be taken into account. Additionally, an equation is included in the code which enables to estimate the lowest natural period of tall multi-story buildings based on their geometrical properties.

$$
T=0.08 \mathrm{~h} / \sqrt{\mathrm{b}_{\mathrm{m}}}
$$

where $b_{m}$ is the mean length of the structure in along wind direction.

Moreover, numerous values of logarithmic damping decrements are summarized in a table listed in the Appendix 2 of the new code. This table as well as the Equ.s 1 and 2 are suitable tools for designers less experienced in wind engineering. Especially at the state of a preliminary design these values can help to provide a rough estimate of the dynamic characteristics and, hence, the dynamic wind loads caused by gust excitation excerted on structures.

In the code OENORM B $4014 / 1$ all types of wind loads are considered which can be described with equivalent quasisteady wind loads excerted on rigid structures. In other words in such cases the wind loads do not cause a significant dynamic response of the building. Generally, in all wind loads codes such quasistatic mechanisms are described and, therefore, the content of this code will not be discussed herein.

However, some parts of the dynamic wind load code OENORM B4014/2 are related to the static wind load code mentioned above, i.e. meteorological information as well as dynamic wind loads due to buffeting are based on reference values determined according to the OENORM B4014/1. This meteorological information mentioned above includes a wind zone map of Austria - reference wind speeds can be obtained from this map or a corresponding table - and a 
classification. of the roughness of the upstream terrain at the construction site. Three types of terrain are considered i.e. open land with very few disturbances such as small houses or trees (type I: surface roughness k in the order of $5.10^{-2} \mathrm{~m}$ ), areas with numerous disturbances such as woods, small towns and suburban areas (type II: $k_{0}$ in the order of $6.10^{-1} \mathrm{~m}$ ) and, finally, urban areas with large scale disturbances (type lII: ko in the order of $3 \cdot 10^{0} \mathrm{~m}$ ).

Furthermore, in the code OENORM B4014/1 valuable information concerning force as well as pressure coefficients for various body shapes, roofs, flags, scaffolds etc. is included which are not explicitly discussed herein.

\section{NEW AUSTRIAN CODE B4014/T2 FOR DYNAMIC WIND LOADS}

Following the idea of separating wind loads on one hand for structures not susceptible and on the other hand susceptible for dynamic wind effects the new code OENORM B4014/2 just concentrates on the latter effects. As mentioned above dynamic wind loads recently gain more importance since structures now can be designed with rather low eigenfrequencies i.e. either very flexible and small or large scale structures with relatively high specific mass densities such as tall buildings or long span bridges. Of course, various types of dynamic wind loadings are of major importance for different groups of stuctures. Consequently, a rough classification of the wind effects to be considered in the design of a particular structure is summarized in a table included in the appendix 1 of the code (see Tab. 1)

Tab. 1: Empirical experiences about structures susceptible for dynamic wind loads

\begin{tabular}{|c|c|c|}
\hline buffeting: & $\begin{array}{l}\text { vortex induced } \\
\text { wind loads: }\end{array}$ & flutter loads: \\
\hline $\begin{array}{l}\text { structures with } \\
\text { either h>100m } \\
\text { or h:b>20 }\end{array}$ & $\begin{array}{l}\text { structures with } \\
\text { circular cross } \\
\text { sections and h: } d>10\end{array}$ & . \\
\hline $\begin{array}{l}\text { buildings with } \\
\text { h }>40 \mathrm{~m} \text { if they } \\
\text { are susceptible to } \\
\text { vibrations accor- } \\
\text { ding to Equ. } 1 \\
\text { - tall buildings } \\
\text { - } \text { TV towers } \\
- \text { observation towers } \\
\text { - light towers } \\
\text { - cooling towers } \\
- \text { chimneys }\end{array}$ & \begin{tabular}{|l} 
- observation towers \\
- light towers \\
- chimneys \\
- constructions and \\
parts of it with \\
circular cross sectio \\
and high slenderness \\
- long span piping \\
systems \\
slender rods
\end{tabular} & $\begin{array}{l}\text { - suspension bridges } \\
\text { - cablestayed " } \\
\text { - girder bridge with } \\
\text { main span length } \\
>150 \mathrm{~m}\end{array}$ \\
\hline
\end{tabular}

It can be seen that three types of dynamic wind loads are taken into i.e. buffeting, vortex induced forces and forces caused by flutter mechanisms. Besides these three wind load processes, of course, other excitation processes caused by wind can also be 
observed such as galloping, interference oscillations, ovalling etc.. However, at the present state of the art a reasonable codification of these latter load mechanisms seems to be rather doubtful since many of the mechanisms involved can only be described with empirical relations. Moreover, a large number of parameters generally has to be taken into account so that a simplified consideration of these mechanisms in a wind load code can lead to incorrect results in particular cases.

In the first section of the new code the meteorological information corresponding to the dynamic wind loads is summarized. This meteorological information includes the height dependent one hour mean wind speeds and stagnation pressures as a function of the terrain category and the reference wind speed. Moreover, an equation for the wind and pressure profile is provided which is based on the power law and enables a simple description of the height dependence of the mean wind speed as well as pressure.

\subsection{Gust Excited Vibrations}

Generally speaking dynamic fluid forces are excerted on all structures subjected to the wind flow in the atmospheric boundary layer. However, only systems with eigenfrequencies sufficently low in the energy containing range of the wind spectrum can be excited to exhibit significant vibrations due to these dynamic forces. Moreover, the vibrations caused by gusts i.e. the turbulent flow in the atmospheric boundary layer mainly occur in along wind direction. Across wind and torsional vibrations, of course, can also be observed even exceeding the amplitudes of along wind response. However, other mechanisms rather than lateral as well as vertical turbulence cause these dynamic responses. Therefore, only along wind gust effects are discussed in the section 2 of the code OENORM B4014/2.

The procedure provided in the code to determine equivalent loads for these dynamic along wind gust loadings is based on the application of statistical concepts in wind engineering as suggested by Davenport (Ref. 4). The application of such concepts is based on the fact that loads induced by buffeting can only be described with quantities such as statistical moments or probability distribution functions because of the random properties of these processes. Consequently, the response processes of structures due to buffeting is characterized by such probabilistic properties, as well. The basic idea summarized in the "Davenport Concept" is to apply the power spectral method in wind engineering in order to determine the r.m.s. values of the along wind response. Based on these statistical quantities extreme value distributions of the response process can be identified which allow the determination of exceedance probabilities of particular response levels. Of course, mean values of the response can simply be computed by applying a standard static analysis.

Finally, in the "Davenport Concept" the method mentioned above has been simplified by considering only one structural mode of major importance and to separate the stochastic response in a resonance and a background part. The basic relations of this 
concept are sketched in the Fig. 2 .
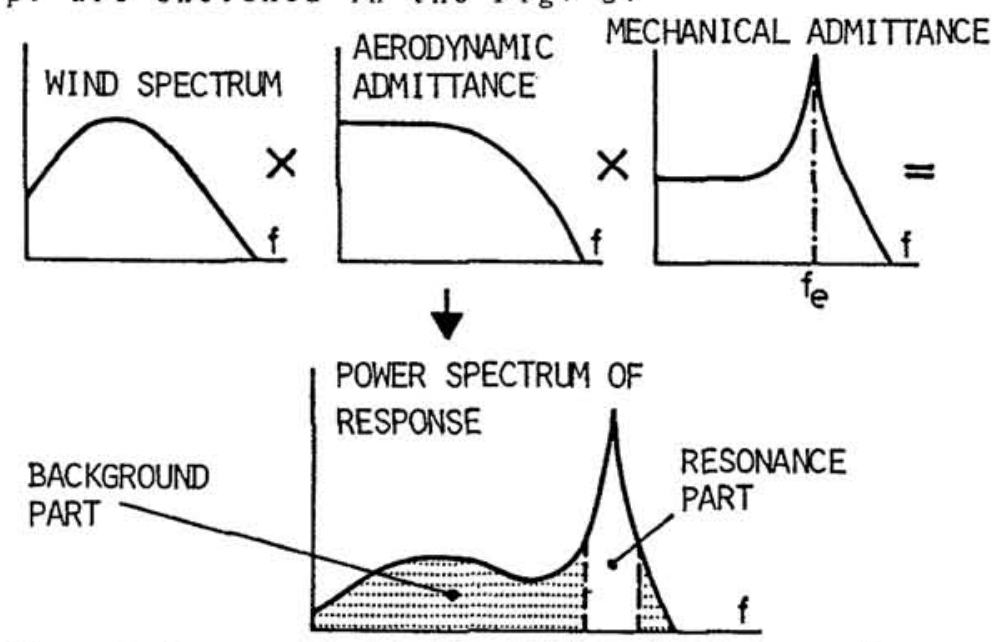

Fig. 2: Davenport Concept for buffeting (Ref. 5)

In a further step of simplification the total equivalent along wind loads including the mean and the dynamic part can be considered with a "gust response factor" (GRF) which relates the mean to an extreme value of the response process with a particular exceedance probability. Of course, by utilizing the GRF which actually is a statistical quantity related to the response process to characterize the ratio of mean and extreme wind loads implicitly includes the assumption of linearity between the input and output process. As mentioned above the extreme value distribution of the response process can approximately be computed based on the corresponding statistical process characteristics i.e. the r.m.s. value and the mean zerocrossing frequency.

The exceedance probability of the particular extreme value to compute the GRF, of course, has to be selected according to the reliability level of the structure required. In Austria a respective reliability code OENORM B4040 just recent Iy has been developed (Ref. 6) in order to balance the risk level of civil engineering structures. Such reliability assessments have to consider the probabilistic properties of the load processes, the type of structure and its technical design as well as its purpose. Obviously, the exceedance probability of the extreme value used for the determination of the GRF should be a function of the parameters mentioned before. However, for the lack of simplicity particular "peak values" gs have been proposed in various codes which simply relate the r.m.s. value of the response or wind load, respectively, to the corresponding extreme value. These values generally vary between 3 . and 4 . (see e.g. Ref. 7-9). Based on the reliability concept of the Austrian code OENORM B4040 mentioned above this peak value is determined with $\mathrm{g}_{\mathrm{S}}=3.6$ assuming a "standard type" tall building which corresponds to a failure probability of the ultimate 1 imit state due to wind loading of $10^{-6}$. Consequently, the equivalent static wind load under consideration of along wind buffeting can be computed as follows: 


$$
\begin{aligned}
& \mathrm{w}_{z}=\varphi \mathrm{c} \overline{\mathrm{q}}_{z} \\
& \varphi=1+\mathrm{g}_{\mathrm{s}} \sigma_{\mathrm{w}} \\
& \sigma_{\mathrm{w}}=4 \mathrm{I}_{\mathrm{h}} \sqrt{\mathrm{s}_{1} \mathrm{~F}_{1} /\left(\delta+\delta_{\mathrm{a}}\right)+\mathrm{B}_{1}} / \sqrt{3} \\
& \delta_{\mathrm{a}}=\rho \mathrm{I}_{\mathrm{m}} \overrightarrow{\mathrm{v}}_{\mathrm{h}} \mathrm{c} / 2 \mathrm{~m}_{\mathrm{G}} \mathrm{f}
\end{aligned}
$$

where $\varphi$ is the GRF, $\sigma_{w}$ the ratio of the r.m.s. and mean value of the wind load, I the turbulence intensity, s the dynamic size factor, $F_{1}$ the gust energy factor, $\delta$ the logarithmic damping decrement of the structure and $\delta_{a}$ the corresponding aerodynamic damping decrement, $B_{1}$ the gust background factor, $I_{m}$ the mean length of the structure perpendicular to the mean wind direction, $\rho$ the air density, $\bar{v}_{h}$ the mean wind speed at the top of the building, fe the first eigenfrequency of the structure (fe $=$ $1 / T), m_{G}$ the generalized mass (Equ. 19) and $c$ the along wind load coefficient of the structure. In Equ. 5 the expression $s_{1} F_{1} /\left(\delta+\delta_{a}\right)$ characterizes the resonance and $B_{1}$ the background part of the dynamic response. All parameters in the Equ.s $3-6$ can be determined based on the properties of the wind at the construction site and the sṭructural characteristics. Both, the related equations and corresponding graphs of these functions are included in the code. These respective equations are shown be low.

$$
\begin{aligned}
& \text { terrain type No: } \\
& \text { I } \\
& \text { II } \\
& \text { I I I } \\
& \begin{array}{l}
\mathrm{I}_{\mathrm{h}}=0.17(10 / \mathrm{h}) 0.15 \\
\mathrm{I}_{\mathrm{h}}=0.26(10 / \mathrm{h}) 0.22 \\
\mathrm{I}_{\mathrm{h}}=0.46(10 / \mathrm{h}) 0.33
\end{array} \\
& \mathrm{~s}_{1}=\pi / 3\left[1+3 \mathrm{f} \mathrm{e}^{\left.\mathrm{h} / \overline{\mathrm{V}}_{\mathrm{h}}\right]\left[1+6 \mathrm{f}^{1} \mathrm{e}_{\mathrm{m}} / \overline{\mathrm{V}}_{\mathrm{h}}\right]}\right.
\end{aligned}
$$

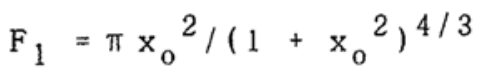

with $x_{0}=1200 \mathrm{f} \mathrm{e}^{/ \overline{\mathrm{V}}_{\mathrm{h}}}$

$$
B_{1}=(2 / 3){ }_{0} f^{900 / h}\left\{x /\left[(1+x h / 400)\left(1+x 1_{m} / 200\right)\left(1+x^{2}\right)^{4 / 3}\right]\right) d x
$$

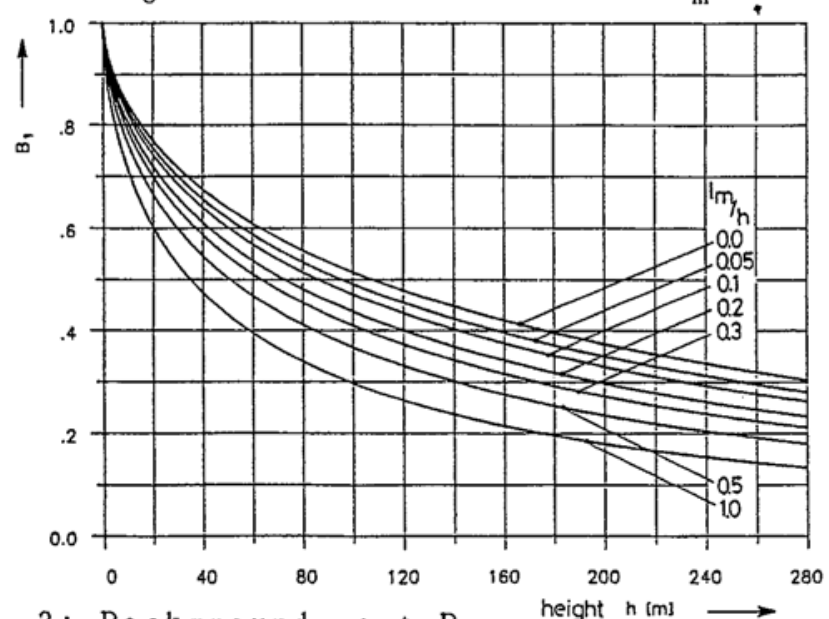

Fig. 3: Background part $B_{1}$ 
It can be seen that this method to consider gust excited response (or wind load, respectively) can simply be included in present design procedures by multipying the wind loads with this GRF. Similar approaches based on the Davenport concept have already previously been included in the other wind load codes such as one of first the Canadian wind load code (Ref. 7).

As a concluding remark it should be mentioned that this procedure leads to good results as long as the assumptions such as linearity, one dominating structural mode and a simple geometrical layout e.g. height independent rectangular cross section are satisfied. However, in particular cases of either significant economical importance or particular reliability requirements wind tunnel tests as well as advanced analytical analyses should be performed to obtain more reliable information about the along wind gust loads.

\subsection{Vortex Excited Vibrations}

Various types of vortex shedding mechanisms can be identified (see e.g. Ref. 10) which cause significant dynamic loads and, consequently, structural vibrations. However, the mechanism of major importance, especially in the case of circular cross sections, is known as Karman-vortex-shedding. Thus, the content of the code OENORM B4014/2 concentrates on the description of this mechanism, only. Moreover, the influence of adjecant buildings which can cause interference effects is not considered. However, in the appendix 3.2 of the code these effects are briefly discussed. A detailed analysis of such load processes requires the application of more advanced analytical procedures and, therefore, only a qualitative characterization of the basic mechanism is provided.
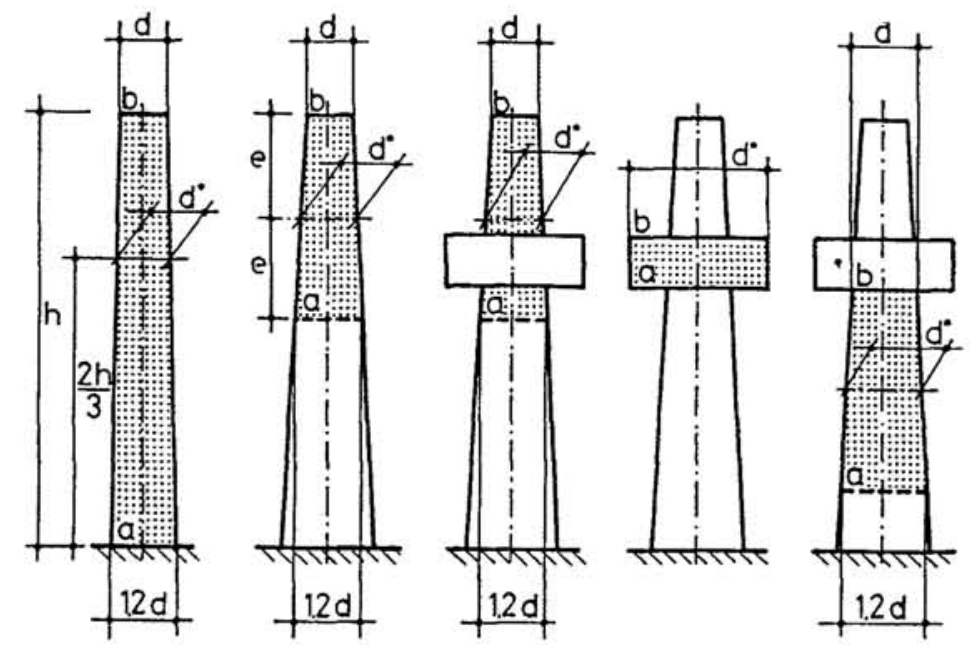

Fig. 4: Vortex loads on tall structures (Ref. 2)

The typical mechanism of Karman vortex shedding can be summarized as an alternating vortex separation at the side faces of a cross section. The frequency of this process is generally dominated by the mean flow velocity and the geometric properties of the cross 
section. Consequently, a nondimensional number - the Strouhal number $S$ - is defined as follows:

$$
\mathrm{S}=\mathrm{f}_{\mathrm{v}} \mathrm{d}^{*} / \overline{\mathrm{V}}
$$

where $f_{v}$ is the Karman vortex shedding frequency and $d^{*}$ the characteristic length of a particular cross section (see Fig. 4). Obviously, resonance effects can occur if one of the eigenfrequencies of a structure fe is close to this Karman vortex shedding frequency $f_{v}$. Based on this fact the following criterion is provided in the code to decide whether or not this effect should be considered.

$$
\mathrm{f}_{\mathrm{e}}>\mathrm{f}_{\mathrm{V}}=\overline{\mathrm{v}}_{\mathrm{h}} \mathrm{S} / \mathrm{d}^{*}
$$

This criterion is based on the lowest eigenfrequency of the structure and, hence, this mechanism has not to be taken into account as long as the corresponding Strouhal frequency $f_{V}$ is less than this lowest eigenfrequency fe.

On the other hand if the structure is identified to be susceptible to vortex induced vibrations a semi-empirical procedure is provided in order to determine the amplitudes of the dynamic deflections of the structure due to the action of Karman vortex shedding. These maximum amplitudes are computed by assuming linear mechanical properties and considering lateral generalized forces based on corresponding lateral force coefficients. In the case of structures with cross section varying with height these loads have only to be considered in particular parts of the structure as shown in Fig. 4.

With the assumptions made above the amplitudes of the structural response due to Karman vortex shedding can be calculated based on generalized lateral forces under consideration of experimentally determined lateral force coefficients. However, by taking into account the results of experimental investigations as summarized in Ref. 11, the analytical expressions are modified in order to consider these results. Hence, the lateral deflections of a structure can be computed with the following set of equations:

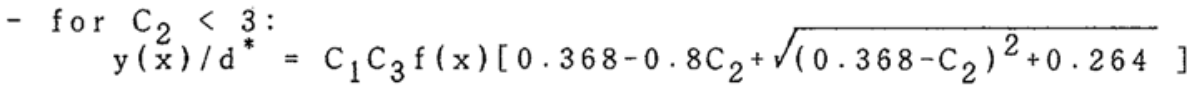

$$
\begin{aligned}
& \text { - for } C_{2} \geqslant 3 \text { : } \\
& y(x) / d^{*}=0.132 C_{1} C_{3} f(x) /\left(0.8 C_{2}-0.368\right) \\
& C_{1}={ }_{a} \int^{b} f(x) d x /{ }_{0} \int^{h} f(x)^{2} d x \\
& \mathrm{C}_{2}=\pi \mathrm{Sc} \mathrm{s} \mathrm{S}^{2}\left(1+0.003 \Lambda \pi \mathrm{Sc} \mathrm{s} \mathrm{s}^{2} / \mathrm{C}_{\mathrm{s}}\right) / \mathrm{C}_{\mathrm{s}} \\
& v_{d}^{*}=f_{e} d^{*} / s \\
& \mathrm{~V}_{\mathrm{d}}^{*} \leqslant 12 \mathrm{~m} / \mathrm{s}: \\
& \begin{array}{ll}
12 \mathrm{~m} / \mathrm{s}<\mathrm{V}_{\mathrm{S}^{*}}^{*} \leqslant 22 \mathrm{~m} / \mathrm{s}: & \mathrm{C}_{3}=1-0.1\left(\mathrm{~V}_{\mathrm{d}^{*}}-12\right) \\
\mathrm{V}_{\mathrm{d}}^{*}>22 \mathrm{~m} / \mathrm{s}: & \mathrm{C}_{3}=0.0
\end{array} \\
& C_{3}=0.0 \\
& \mathrm{Sc}=2 \mathrm{~m}_{\mathrm{G}} \delta / \rho \mathrm{d}^{* 2}
\end{aligned}
$$




$$
\begin{aligned}
& { }_{m_{G}}={ }_{0} \int^{h}(x) f(x)^{2} d x / \int_{0} f_{f(x)}{ }^{2} d x \\
& \Lambda=(b-a) / d^{*}
\end{aligned}
$$

where $y(x)$ is the lateral deflection as a function of the height $x$ above ground, $f(x)$ the mode shape, $m(x)$ the mass per length along the height of the structure and a as well as b are the limits of the section to be considered (see Fig. 4).

The lateral force coefficient $C_{s}$ in Equ. 15, of course, depends on the geometrical shape of the cross section as well as on flow related parameters such as the mean wind speed, the turbulence scale as well as -intensity. However, the influence of the latter two parameters is frequently neglected, especially in the case of circular cross sections. Consequently, this lateral force coefficient can be described as a function of the Reynolds number. Such a plot is also included in the code (see Fig. 5).

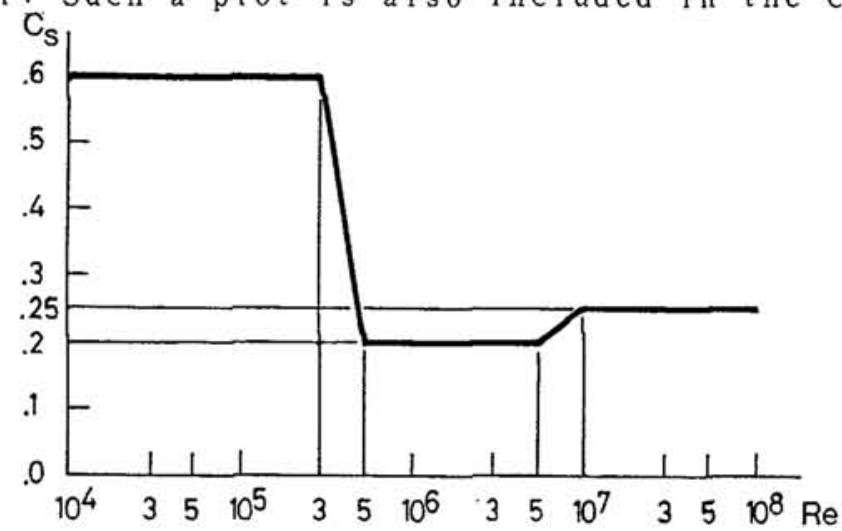

Fig. 5: Lateral force coefficient for circular cross section (Ref. 2)

\subsection{Flutter Vibrations}

Similarly, as in the case of vortex induced vibrations, various mechanisms of flutter can be identified where torsional-and coupled-(torsional-bending)flutter are the load processes of major importance especially in bridge engineering (Ref. 10). The latter process has to be considered if the eigenfrequencies of the bending mode perpendicular to the mean wind direction and the torsional mode are very close.

Again, a critical quantity can be defined to decide whether or not flutter mechanisms have to be considered. In this case a critical wind speed based on the frequency characteristics of the structure is defined which marks the upper stability bound. In other words if the mean wind speed $V_{h}$ obtained from meteorological data in this code exceeds this critical value $V_{k}$ fluter vibrations are likely to occur.

$$
\mathrm{v}_{\mathrm{k}} \leqslant \overline{\mathrm{v}}_{\mathrm{h}}
$$

The equation to determine this critical wind speed is based on a procedure suggested by Kloeppel and Thiele (Ref. 12) and modified by ECCS (Ref, 13). 


$$
v_{k}=k \pi f_{e}, b b\left[1+\left(f_{e}, t / f_{e}, b-0.5\right) \sqrt{1.5 m i / \rho b^{3}}\right]
$$

where $f e, b$ and $f e, t$ are the bending- as well as torsional eigenfrequency of the structure of major importance, i is the polar radius of inertia and b the length of the structure in along wind direction.

The empirical factor k generally has to be determined in wind tunnel tests. However, for a first estimation of the flutter stability of a structure e.g. during a preliminary design this factor can be adopted from similar investigations. The results of such analysis are also summarized in Ref. 12 and parts of the respective tables are included in the code (see Tab. 2).

If the criterion above indicates flutter instabilities likely to occur either the structural design (geometric and structural properties) has to be revised or detailed investigations of the flutter characteristcs of the structure have to be performed (e.g. wind tunnel tests). However, this complex procedure cannot be included in such a wind load code because too many parameters are to be considered in such a design process.

Tab. 2: Factor k for various cross sections (Ref. 2)

\begin{tabular}{|c|c|c|c|c|c|}
\hline$\frac{h}{b}$ & $\operatorname{lot}_{+}^{\frac{1}{1}}$ & $\prod_{-b \rightarrow+}^{\frac{1}{f}}$ & $\begin{array}{l}2 b \leqslant x \leqslant 7 b+1 \\
+x++1+1\end{array}$ & $\begin{array}{l}2 b \leq x \leq 7 b \text { । } \\
+x+1+1 \\
+b+1\end{array}$ & $\stackrel{\rightleftarrows_{t b-t}}{\rightleftharpoons}$ \\
\hline .05 & .40 & .30 & - & - & .70 \\
\hline .10 & .15 & .12 & .50 & .80 & .70 \\
\hline .15 & 12 & .11 & .50 & .70 & .70 \\
\hline .20 & .10 & .10 & - & - & - \\
\hline .30 & - & - & .25 & .30 & - \\
\hline
\end{tabular}

\section{4 Appendecies}

In the first appendix some examples of structures are summarized which generally are susceptible to either one of the dynamic wind load mechanisms discussed above. The content of this table is a l ready shown in Tab. 1 .

In order to determine the GRF or the $S$ number logarithmic damping decrements have to be known for the system under consideration. However, an analytical computation of such damping characteristics based on the structural design can still not be performed at the present state of the art. Because of this, in the appendix 2 various damping decrements for materials, structural design conditions as well as foundations and supportings are summarized.

In the last appendix two special effects of wind induced loads are discussed briefly i.e. the galloping mechanism and interference effects. The latter process has already been mentioned before. The galloping mechanism is caused by the interaction of the wind flow and the structural motion. Again, as 
already shown for the flutter process a critical wind speed can be defined which is based on the Den Hartog criterion by considering the stuctural and aerodynamic damping which actually is in this case negative. In other words, energy is transferred from the flow to the structure due the structural motion.

$$
\mathrm{V}_{\mathrm{k}}=\left(\begin{array}{ll}
\mathrm{m} \delta \mathrm{f} & \mathrm{e} / \rho \mathrm{l}_{\mathrm{m}}
\end{array}\right) \mathrm{c}
$$

where the structure is considered to be susceptible for galloping vibrations if

$$
\overline{\mathrm{v}}_{\mathrm{h}} \geqslant \mathrm{v}_{\mathrm{k}}
$$

The factor c in the Equ. 21 is related to the derivative of the lift force coefficient of the cross section and has to determined experimentally. The respective values for rectangular cross section with bluffness ratios of $0.5,1.0$ and 2.0 are included in the appendix. However, it should be mentioned that this approach is based on linear theory.

There is a strong influence of turbulence on these values, therefore one has to be careful in taking values from the literature, because most of them depend on smooth flow. Thus, the Equ. 22 just provides a rough assessment of the galloping stability of a structure. For detailed analyses wind tunnel test might be performed.

\section{NUMER ICAL EXAMPLES}

The application of this new wind load code will be demonstrated by analyzing two particular structures i.e. a ta!l RC building and a steel chimney. In both cases the structure is assumed to be located in the city of Vienna which leads to urban area characteristics (type Ill terrain). The power law exponent of the wind profil is due to OENORM B4014/2 $\alpha=0.33$. Either from the wind zone map or the respective table, a corresponding reference wind speed of $140 \mathrm{~km} / \mathrm{h}$ can be determined. Based on this reference value the one hourly mean wind speed at the height of $100 \mathrm{~m}$ can be found with $27.7 \mathrm{~m} / \mathrm{s}$ and stagnation pressure profil can be obtained from the corresponding equation.

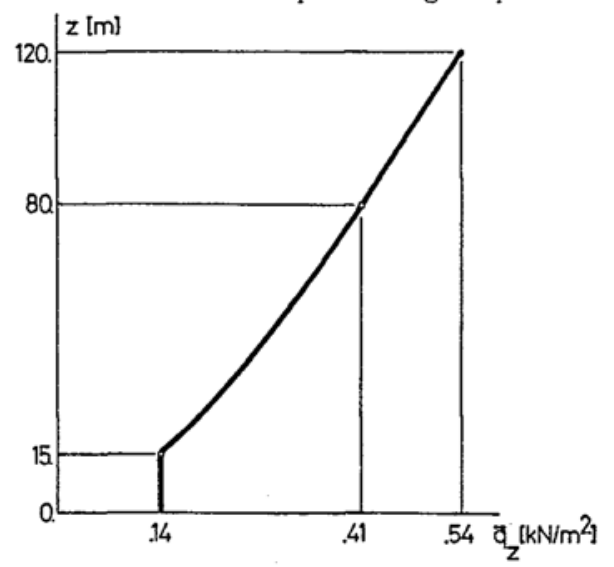

Fig. 6: Wind pressure profil at the location Vienna 


\section{Example 1:}

The first example is representative for a class of structures such as tall buildings in urban areas. The geometric as well as structural properties of the building are defined in Fig. 7 . Based on these input data the equivalent along wind loads due to buffeting can be determined as follows:

- determination of the natural period of the bending mode in along wind direction:

(Equ. 2) $\quad \mathrm{T}=0.08 \mathrm{~h} / \sqrt{\mathrm{b}_{\mathrm{m}}}=1.92 \mathrm{~s}$

$$
\mathrm{f}_{\mathrm{e}}=1 / \mathrm{T}=0.52 \mathrm{~Hz}
$$

- logarithmic damping decrement (OENORM B4014/2, Appendix 2):

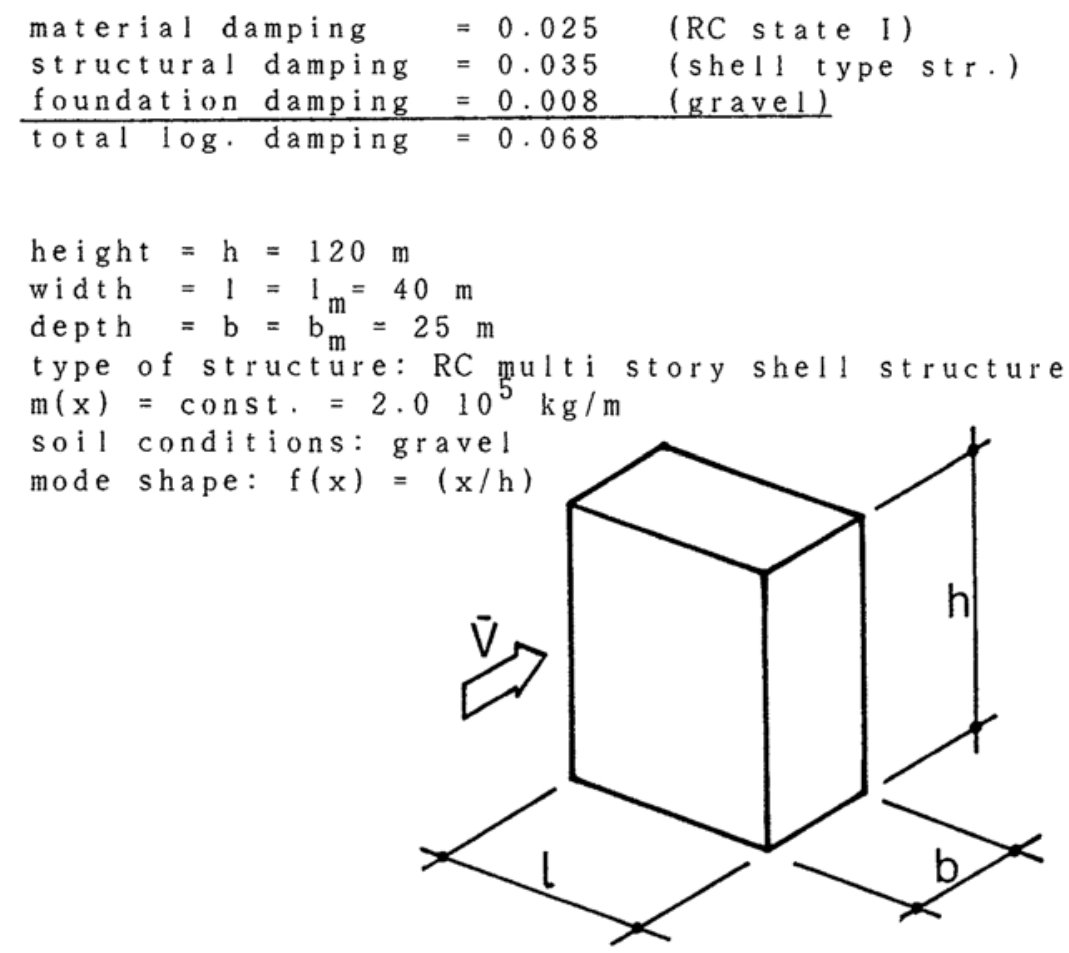

Fig. 7: Sample structure of the first example

According to OENORM B $4040 / 2$, dynamic wind loads generally have to be considered for structures with a height greater than $100 \mathrm{~m}$. As it will be shown 1 ater this is a realistic assumption since these dynamic effects significantly contribute to the total wind loads in almost all of such cases.

- mean wind speed at the top of the building (OENORM B4014/2, Equ. 1):

$$
\overline{\mathrm{V}}_{\mathrm{h}}=\mathrm{V}_{100}(\mathrm{~h} / 100)^{0.33}=29.4 \mathrm{~m} / \mathrm{s}
$$


- drag force coefficient (OENORM B4014/1, Tab. 7):

$$
c(h / 1=3.0,1 / b=1.6)=1.38
$$

- gust response factor (GRF):

(Equ. $7 \mathrm{c}) I_{\mathrm{h}}=0.46(10 / \mathrm{h})^{0.33}=0.20$

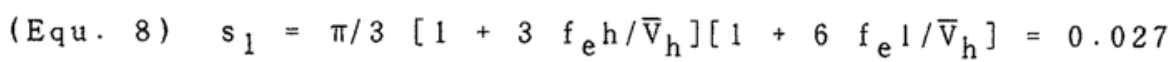

(Equ. 9) $\quad F_{1}=\pi x_{0}{ }^{2} /\left(1+x_{0}{ }^{2}\right)^{4 / 3}=0.4086$

with $x_{o}=1200 \mathrm{f}_{\mathrm{e}} / \overline{\mathrm{V}}_{\mathrm{h}}$

(Equ. 10, Fig. 3) $B_{1}(\mathrm{~h}=120 \cdot \mathrm{m}, 1 / \mathrm{h}=0.33)=0.40$

Since the mass density is independent of the height it follows:

(Equ. 19) $m_{G}={ }_{0} \int^{h} m(x) f^{2}(x) d x / \int_{0}^{h} f^{2}(x) d x=m$

(Equ. 6) $\quad \delta_{\mathrm{a}}=\rho \mathrm{m}_{\mathrm{m}} \mathrm{V}_{\mathrm{h}} \mathrm{c} / 2 \mathrm{~m}_{\mathrm{G}} \mathrm{f}_{\mathrm{e}}=0.01$

(Equ. 5) $\quad \sigma_{\mathrm{w}}=4 \mathrm{I}_{\mathrm{h}} \sqrt{\mathrm{s}_{1} \mathrm{~F}_{1} /\left(\delta+\delta_{\mathrm{a}}\right)+\mathrm{B}_{1}} / \sqrt{3}$

$$
=40.20 \sqrt{0.14+0.40} / \sqrt{3}=0.34
$$

From the result above it can be seen that in the example analyzed the resonance part contributes already $25 \%$ to the total dynamic response. Finally, the GRF can be determined.

(Equ. 4) $\varphi=1+\mathrm{g}_{\mathrm{S}} \sigma_{\mathrm{W}}=1+3.60 .34=2.22$

The equivalent along wind loads under consideration of buffeting effects are shown in the figure below.

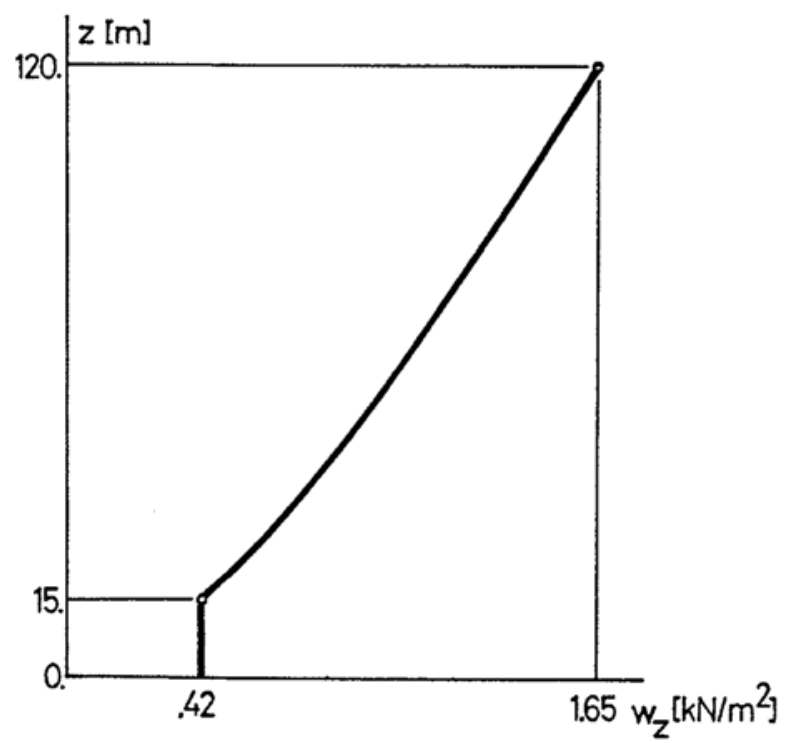

Fig. 8: Equivalent along wind forces for example 1 
In a further step the susceptibility of the structure to vortex induced vibrations should be investigated. By applying Equ. 12 a structure is defined not to be susceptible if

(Equ. 12) $\mathrm{f}_{\mathrm{e}}>\overline{\mathrm{V}}_{\mathrm{h}} \mathrm{S} / \mathrm{d}_{*}$

$$
\mathrm{d}_{*}=1=40 \cdot \mathrm{m}
$$

From literature the value for the Strouhal number $S$ can be found with 0.13 for a bluffness ratio of 2 . It can be seen that the inequality above $(0.52>0.096)$ is sat isfied and, consequently, the structure can be assumed not to be susceptible to vortex induced vibrations.

Expample 2:

In the second example a tall steel chimney as sketched in Fig. 9 will be analyzed. Again, it is assumed, that the construction site is Vienna. Hence, the same meteorological input data can be utilized as in the first example.

In a first step, the susceptibility of the structure for dynamic wind loads has to be analyzed.
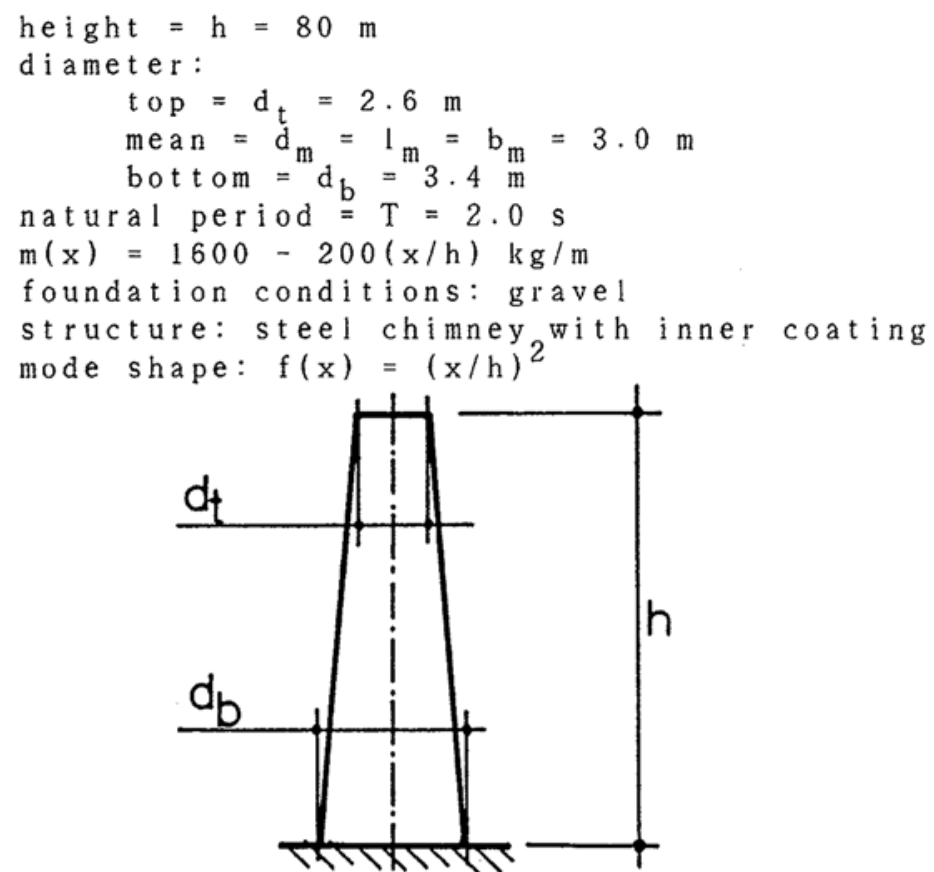

Fig. 9: Sample structure of the second example

- logarithmic damping decrement (OENORM B4014/2, Appendix 2):

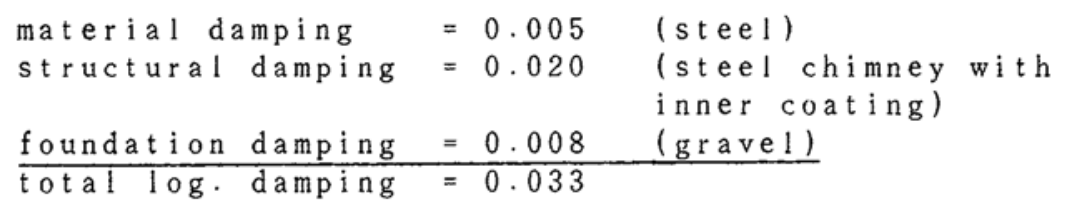


- susceptibility factor for dynamic wind effects:

$\left(\right.$ Equ. 1) $f=T\left(v_{0} / h\right)\left(h / 1_{m}\right)^{1 / 3}\left(\mathrm{~h} / h_{0}\right)^{1 / 7}=2.54$

Considering the damping decrement and the factor $f$ it can be concluded from the nomogram (Fig. 1) that the effect of dynamic wind loads has to be considered for this structure. Consequently, the equivalent along wind loads have to be determined by taking into account the GRF which can be computed as follows.

- mean wind speed at the top of the structure (OENORM B4014/2, Equ. 1):

$$
\bar{v}_{h}=v_{100}(80 / 100)^{0.33}=25.7 \mathrm{~m} / \mathrm{s}
$$

- drag force coefficient for a smooth surface $(k=2 \mathrm{~mm}$, OENORM B4014/1, Fig. 13 and 14):

$$
\begin{aligned}
& c_{z}\left(d \sqrt{q_{h}}=1.67, k / d=2 / 3000=10^{-2}\right)=0.95 \\
& k_{1}(\Lambda=h / d=26.67)=0.85 \\
& c=k_{1} c_{z}=0.850 .95=0.81
\end{aligned}
$$

- gust response factor (GRF):

$($ Equ. $7 \mathrm{c}) \mathrm{I}_{\mathrm{h}}=0.46(10 / \mathrm{h})^{0.33}=0.23$

(Equ. 8) $\mathrm{s}_{1}=\pi / 3\left[1+3 \mathrm{f}_{\mathrm{e}} \mathrm{h} / \overline{\mathrm{V}}_{\mathrm{h}}\right]\left[1+6 \mathrm{f}_{\mathrm{e}} \mathrm{l} / \overline{\mathrm{V}}_{\mathrm{h}}\right]=0.137$

(Equ. 9) $\quad F_{1}=\pi x_{0}{ }^{2} /\left(1+x_{0}{ }^{2}\right)^{4 / 3}=0.384$

with $x_{0}=1200 \mathrm{f}_{\mathrm{e}} / \overline{\mathrm{V}}_{\mathrm{h}}$

(Equ. 10, Fig. 3) $B_{1}(h=80 \cdot m, d / h=0.037)=0.54$

(Equ. 19) $m_{G}={ }_{0} \int^{h} m(x) f^{2}(x) d x /{ }_{0} \int^{h} f^{2}(x) d x=1433.3 \mathrm{~kg} / \mathrm{m}$

(Equ. 6) $\delta_{\mathrm{a}}=\rho \mathrm{m}_{\mathrm{m}} \mathrm{V}_{\mathrm{h}} \mathrm{c} / 2 \mathrm{~m}_{\mathrm{G}}^{\mathrm{f}} \mathrm{e}=0.054$

(Equ. 5) $\quad \sigma_{\mathrm{w}}=4 \mathrm{I}_{\mathrm{h}} \sqrt{\mathrm{s}_{1} \mathrm{~F}_{1} /\left(\delta+\delta_{\mathrm{a}}\right)+\mathrm{B}_{1}} / \sqrt{3}$

$$
=40.23 \sqrt{0.605+0.54} / \sqrt{3}=0.57
$$

It can be seen that in this case the resonance part of the dynamic response due to buffeting al ready exceeds the background part. The corresponding GRF can be determined as follows:

$\left(\right.$ Equ. 4) $\varphi=1+\mathrm{g}_{\mathrm{S}} \sigma_{\mathrm{W}}=1+\mathrm{g}_{\mathrm{S}} 0.57=3.05$

The eqivalent along wind loads based on the one hour mean wind pressure and the above GRF are shown in Fig. 10.

In a second step the susceptibility of the structure to vortex induced vibrations has to be investigated by applying the respective criterion as defined in Equ. 12. 


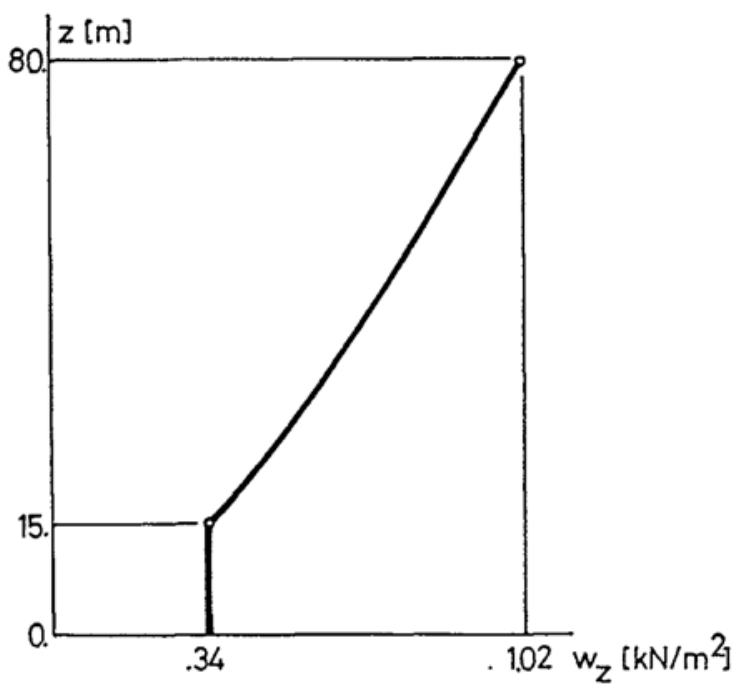

Fig. 10: Equivalent along wind forces of example 2

(Equ. 12) $\mathrm{f}_{\mathrm{e}}=0.50>\overline{\mathrm{v}}_{\mathrm{h}} \mathrm{S} / \mathrm{d}^{*}=1.80$

where the charateristic diameter $d^{*}$ can be determined as shown in Fig. 11 .

Since the inequality above is not satisfied, vortex induced vibrations have to be considered in the structural analysis. According to the procedure suggested in the new Austrian wind code OENORM B4014/2 the amplitudes due to this dynamic excitation can be calculated as shown in section 3.2 of this paper. Based on these response characteristics related quantities such as load effects easily can be determined.

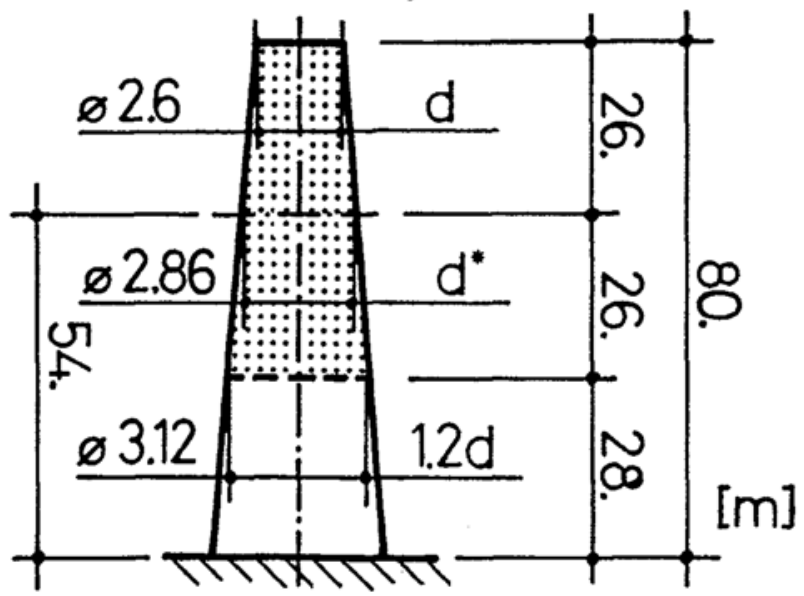

Fig. 11: Determination of the characteristic diameter d $^{*}$

Note, that the lateral forces causing the vortex induced vibrations have only to be considered in the range where the characteristic length of the structure - in this case the diameter - does not vary with more than $20 \%$ as sketched in Fig. 11 . 
- deflections due to vortex induced vibrations:

$\left(\right.$ Equ. 13b) $y(x) / d^{*}=0.132 C_{1} C_{3} f(x) /\left(0.8 C_{2}-0.368\right)$

(Equ. 14) $c_{1}={ }_{a} \int^{b} f(x) d x / 0 f^{h} f(x)^{2} d x=1.60$

(Equ. 18$) \mathrm{Sc}=2 \mathrm{~m}_{\mathrm{G}} \delta / \rho \mathrm{d}^{* 2}=9.25$

(OENORM B4014/2, Equ. 2) $\mathrm{q}_{z}(\mathrm{z}=54 \mathrm{~m})=\mathrm{q}_{100}(\mathrm{z} / 100)^{0.66}=0.32 \mathrm{kN} / \mathrm{m}^{2}$

(OENORM B4014/1, Equ. 16) $\mathrm{Re}=2.6710^{6} \mathrm{~d}^{*} \sqrt{\mathrm{q}_{z}}=4.310^{6}$

(Fig. 5 ) $\quad \mathrm{C}_{\mathrm{S}}=0.2$

(OENORM B4014/2, section 3.2 .1 ) $\mathrm{S}=0.2$

(Equ. 20$) \Lambda=(b-a) / d^{*}=18.2$

(Equ. 15$) \mathrm{C}_{2}=\pi \mathrm{Sc} \mathrm{S}{ }^{2}\left(1+0.003 \Lambda \pi \mathrm{Sc} \mathrm{s} / \mathrm{C}_{\mathrm{s}}\right) / \mathrm{C}_{\mathrm{s}}=7.66$

(Equ. 16$) v_{d^{*}}=f_{e^{d}} / S=7.15$

(Equ. $17 \mathrm{a}) \mathrm{C}_{3}=1.0$

$$
\begin{aligned}
& y(x=h) / d^{*}=y_{\max } / d^{*}=0.0367 \\
& y_{\max }=1.0510^{-1} \mathrm{~m}
\end{aligned}
$$

As already mentioned above from this amplitude of lateral deflections due to vortex induced vibrations related quantities such as bending moments and shear forces can be determined.

\section{COMPARISON WITH PRESENT JAPANESE WIND LOAD CODE}

A comparative study of the design wind loads is performed for the two sample structures analyzed above by considering the Japanese wind load code presently in use (Ref. 14). In this code a similar approach based on the Davenport Concept is provided with respect to along wind buffeting loads as it is suggested in in the new Austrian wind code as well.

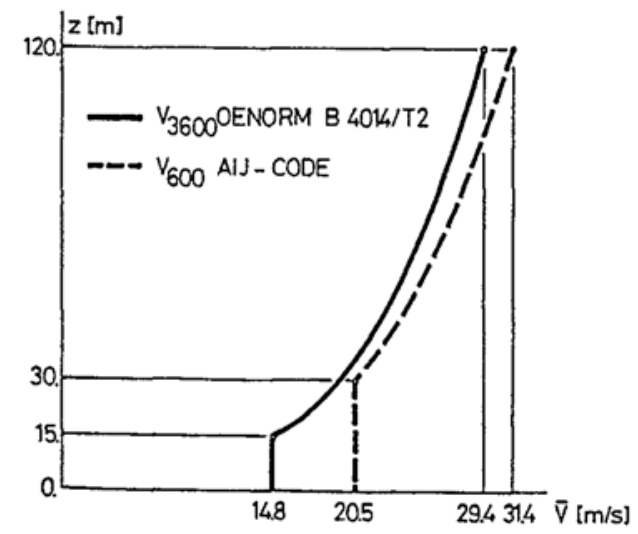

Fig. 12: 10-min and 1 hour mean wind profil 
In the Japanese code annual extreme mean wind speeds are defined on the basis of $10-m i n$ mean values at the height of $10 \mathrm{~m}$ above ground with a return period of 50 years which corresponds to a annual exceedance probability of 0.02 . Hence, in order to determine the wind profil corresponding to the wind speeds defined in the Austrian code, the ratio of 10-min and 1 -hour mean wind speeds has to be taken into account. In Ref. 8 this ratio is shown to be approximately 1.07. Considering the 1-hour mean wind speed at the top of the first sample structure, the respective $10-\mathrm{min}$ mean value computes with $31.4 \mathrm{~m} / \mathrm{s}$. Based on this value the 10-min mean wind profil is computed for the following calculations according to the AlJ-code. This profil along with the 1-hour mean wind profil defined in the OENORM B4014/2 is shown in Fig. 12 .

Following the procedure suggested in AIJ-code the gust response factor $G_{f}$ can be computed as follows:

$$
\begin{aligned}
& G_{f}=1+g_{f} r_{f} \sqrt{B_{f}+R_{f}} \\
& g_{f}=\sqrt{2 \ln \left(600 v_{f}\right)}+0.577 / \sqrt{2 \ln \left(600 v_{f}\right)} \\
& v_{f}=f_{e} \sqrt{R_{f} /\left(B_{f}+R_{f}\right)} \\
& R_{f}=2 \pi S_{f} F / \delta
\end{aligned}
$$

From the tables and figures included in the AIJ-code the following numerical values can be identified by considering the structural data of the first sample structure: $\mathrm{r}_{\mathrm{f}}=0.318, \mathrm{~B}_{\mathrm{f}}=$ $0.58, S_{f}=0.035, F=0.06, \nu_{f}=0.26 ;$ hence, $R_{f}=0.194$ and the peak factor $g_{f}=3.36$. Finally, $G_{f}$ computes with 1.94 . The force coefficient can be calculated with 1.4 so that the profil of the equivalent along wind loads is obtained as shown in Fig. 13. In this figure the wind load profil determined according to the new Austrian code OENORM B4014/2 is plotted as well. A good agreement of the wind loads obtained from both codes can be noticed.

A determination of the equivalent along wind loads for the second sample structure leads to the following values: $g_{f}=3.50, \mathrm{r}_{\mathrm{f}}=$ $0.359, B_{f}=0.70$ and $R_{f}=1.47$. The GRF computes with 2.85 and by considering the force coefficient of 0.7 for circular cross sections with a smooth surface the equivalent wind load profil for the second sample structure can be determined as shown in Fig. 14 .

Again, a good agreement of the equivalent wind loads obtained from both codes can be noticed. In a final step, the GRF for the first sample structure obtained from the OENORM B4014/2 and the AIJ-code is compared with corresponding values of the Canadian code (Ref, 7), ECCS-wind load standard (Ref. 13) and the draft of the new German wind load code DIN 1055/4 (Ref. 9). However, by comparing the GRF's the fact has to be considered that 10.min mean values for the wind speed as well as -pressure are utilized in the AIJ-code, the ECCS standard and the DIN code. Hence, this fact can be taken into account by multiplying these GRF 600 values with the ratio of $10-m i n$ and 1 -hour mean wind pressures which is actually the squared ratio of the respective wind speeds.

Generally, the equations to compute the GRF can be reduced to the 
following form:

$$
\mathrm{GRF}_{3600}=1+\mathrm{g} \mathrm{I} \sqrt{\mathrm{B}+\mathrm{R}}
$$

where $g$ is the peak factor, 1 is a factor proportional to the wind turbulence, B the background factor and $R$ the resonance factor. In the case of the codes using 10-min mean values the $\mathrm{GRF}_{3600}$ can be computed as follows:

$$
\mathrm{GRF}_{3600}=1.07^{2} \mathrm{GRF}_{600}
$$

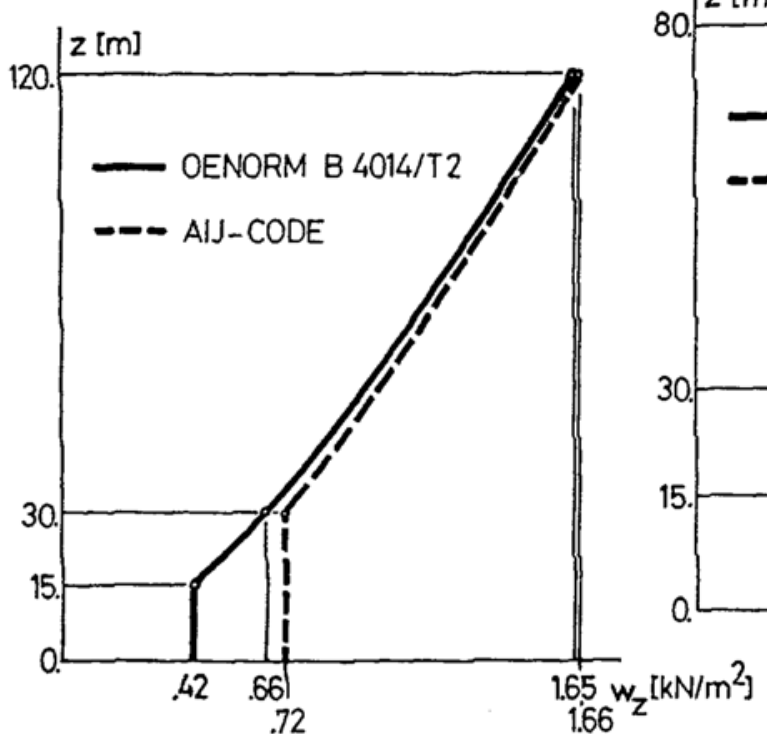

Fig. 13: Equivalent along wind force profil for the first sample structure

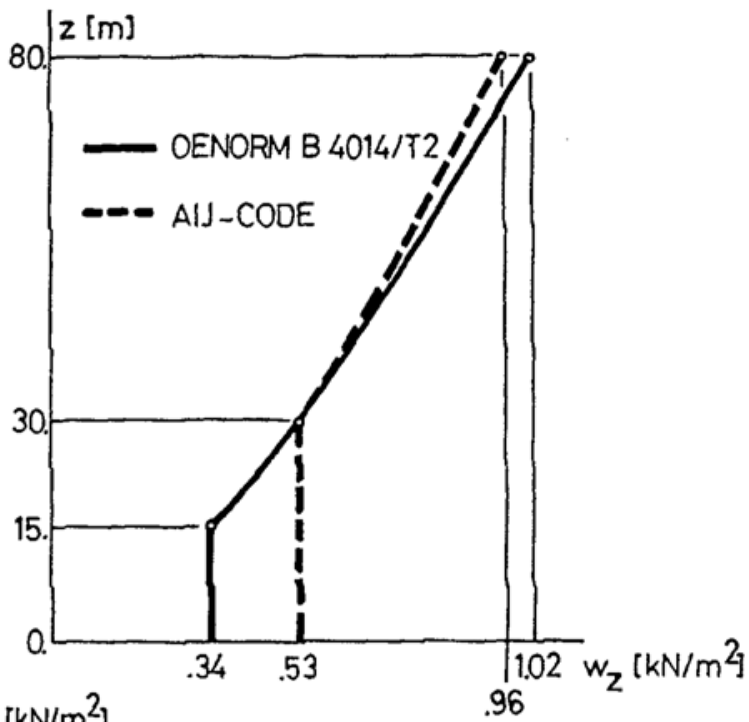

Fig. 14: Equivalent along wind force profil for the second sample structure

Tab. 3: Comparison of the GRF obtained from various wind codes

\begin{tabular}{|l|l|l|l|}
\hline Code & g & GRF $_{3600}$ & $R /(B+R)$ \\
\hline OENORM B4014/T2 & 3.6 & 2.22 & 0.25 \\
AI J of Canada & 3.36 & 2.22 & 0.25 \\
NBC Of & 3.85 & 2.34 & 0.26 \\
DIN 1055/4 & 3.5 & 1.85 & 0.29 \\
ECCS & - & 2.08 & 0.10 \\
\hline
\end{tabular}

From the results presented in Tab. 3 a good agreement of the GRF's can be noticed except the very small value for the GRF calculated according to the DIN 1055/4. This is mainly caused by the fact that the DIN $1055 / 4$ is the only code which does not explicitly consider the influence of the terrain category. Consequently, the reliability level of structures designed according to this DSN code can significantly vary at different construction sites. Moreover, it can be noticed that the dynamic portion of the structural response characterized by the ratio $R /(B+R)$ is approximately the same for all codes except the ECCS 
code provision.

\section{CONCLUSIONS AND PREVIEW ON FURTHER DEVELOPMENT OF WIND LOAD CODIFICATION IN AUSTRIA}

The rules given in codes for wind loads have to be simple for application on the one hand, on the other hand they have to model the loads as realistic as possible. Therefore, a compromise has to be found always.

For the gust excitation the mathematical model presented by Davenport is in agreement with the assumptions given above, therefore it is included in many codes. Though the parameters used in different codes are different, the gust response factors calculated according to these are in rather good agreement as shown in this paper. The same is valid for the ratios of the dynamic part of the load to the sum of dynamic plus quasisteady portions.

The mathematical concept for vortex excited vibrations in the OENORM B4014/2 is simple to apply as shown in the example of the paper. It takes into account that the amplitudes are limited which is in agreement with experience.

For flutter vibrations of bridges, the method suggested by Kloeppel-Thiele commonly used in Europe is presented in a simple form for a check of a problem. If the bridge turns out to be unstable due to these rules, an investigation in more detail has to be started.

For galloping vibrations the Den Hartog criterion is presented in the appendix of the code because there is a lack of reliable data on instability factors c in the literature. More over this, the criterion is based on a linear theory and, therefore, the calculated instability boundary could signficantly deviate from model test-as well as full scale observations.

Now one has to wait for the reaction of the practical engineers to the new rules of OENORM B4014/2. On the other hand, concerning the first part of the new Austrian wind code, the Austrian Codification Institute received several comments since the publication of the OENORM B4014/1 in 1980. Consequently, the revision of this part will start this year.

\section{ACKNOWLEDEGEMENT}

The financial support from the Japanese ministry of education (Monbusho) enabling P.H.W. Prenninger to study at Kyoto University within the International Course $1987 / 89$ is gratefully acknowledged. Furthermore, P.H.W. Prenninger wishes to thank N. Shiraishi and M. Matsumoto for their invitation to work at the Bridge Laboratory of Kyoto University and for their most valuable comments during the course of this work. Special thanks are also due to H. Shirato and A. Mizawa for their help concerning the wind load calculations according to the Japanese code.

\section{REFERNCES}

1 OENORM B4014/T1: Statische Windkraefte, Austrian Codification Institute, Vienna, Austria, 1980 (in German) 
OENORM B4014/T2: Windwirkungen an schwingungsanfaelligen Bauwerken, Austrian Codification Institute, Vienna, Austria, draft March 1988 (in German)

3 Nieser, H.: personal communication

4 Davenport, A.G.: Gust loading Factors, Journ. Struct. Div., ASCE, Vol. 93, ST 3, 1967, pp. 11-34

5 Socke1, H.: Aerodynamik der Bauwerke, Vieweg 1984,412 p, ( i n German)

6 OENORM B4040: Sicherheitskonzept im Bauwesen, Austrian Codification Institute, Vienna, Austria, draft October 1987 (in German)

7 NRCC, 1970a: National Building Code of Canada, 1970, and NRCC 1970b: The Supplement to the National Building Code of Canada, Associate Committee on the National Building Code, National Research Council of Canada, Ottawa, Canada

8 ANSI, 1982: Minimum Design Loads for Buildings and Other Structures, ANSI A58.1-1982, American National Standards Institute, New York

9 DiN 1055/4: Windwirkungen auf Bauwerke, NABau committee 11 $3 / 6$ "Lastannahmen; Windlast", draft April 1986 (in German)

10 Matsumoto, M. : Bridge Aerodynamics and Wind-Resistant Design, Kyoto Univ, and KAIST Joint Meeting 1988, Proc., Ed. N. Shiraishi and C.K. Choi, Jan. 22-23, 1988, Seoul, Korea

11 Blevins, R.D.: Flow-induced Vibrations, Van Nostrand Reinhold Comp., New York 1977, 363 p

12 Kloeppel-Thiele: Modellversuch im Windkanal zur Bemessung von Bruecken gegen die Gefahr winderregter Schwingungen, Stahlbau 36, Heft 12, Dec. 1967 (in German)

13 ECCS: Recommendations for Calculating the Effects of Wind on Constructions, $1 \mathrm{st}$ and 2nd Edition., ECCS-Technical Committee 12-Wind, Report No.52, Brussels, 1978 and 1987

14 Murota, T.: On the Wind Load Proposed by AlJ in "Load Recommendation for Building Design", Journ. of Wind Engineering, No, 12, Apri1, 1982, pp 45-56 